\begin{tabular}{|c|c|c|c|c|}
\hline & JNU & jnul2169 & Dispatch: September 22, 2015 & CE: N/A \\
\hline & Journal & MSP No. & No. of pages: 7 & PE: Matt Scanlon \\
\hline
\end{tabular}

\title{
A Program of Nurse Management for Unscheduled Consultations of Children With Acute Minor Illnesses in Primary Care
}

\author{
Núria Fabrellas, PhD, BA(hons), MSc, Pediatric Nurse Specialist, RN ${ }^{1}$, Eulàlia Juvé, PhD, MSn, RN², \\ Montserrat Solà, PhD, MSn, RN³ , Eva Aurín, BSc ${ }^{4}$, Sofia Berlanga, PhD, BSc, RN ${ }^{5}$, Jordi Galimany, PhD, MSn, \\ $\mathrm{RN}^{6}$, Lidia Berenguer, $\mathrm{RN}^{7}, \mathrm{M}$ Cèlia Pujol, $\mathrm{RN}^{8}$, Sara Lacuesta, $\mathrm{RN}^{9}, \mathrm{M}$ Cinta Villo, $\mathrm{RN}^{10}$, \& Montserrat Torres, \\ $\mathrm{RN}^{11}$
}

1 Professor of Nursing, School of Nursing, University of Barcelona, Barcelona, Catalonia, Spain 2 Nurse Coordinator, Institut Català de la Salut, Associate Professor of Nursing, School of Nursing, University of Barcelona. Barcelona, Catalunya, Spain 3 Professor of Nursing, School of Nursing, University of Barcelona, Barcelona, Catalonia, Spain 4 Computer Scientist, Institut Català de la Salut, Barcelona, Catalunya, Spain

5 Pediatrics care nurse, Institut Català de la Salut, Associate Professor of Nursing, School of Nursing, University of Barcelona, Barcelona, Catalunya, Spain 6 Professor of Nursing, School of Nursing, University of Barcelona, Barcelona, Catalonia, Spain 7 Pediatrics care nurse, SAP Girona, Institut Català de la Salut, Barcelona, Catalunya, Spain 8 Pediatrics care nurse, SAP Alt Penedes, Institut Català de la Salut, Barcelona, Catalunya, Spain 9 Pediatrics care nurse, SAP Terres del Ebre, Institut Català de la Salut, Barcelona, Catalunya, Spain 10 Pediatrics care nurse, SAP Terres del Ebre, Institut Català de la Salut, Barcelona, Catalunya, Spain 11 SAP Delta del Llobregat, Institut Català de la Salut, Barcelona, Catalunya, Spain

Key words

Acute minor illness, pediatric care, primary care

\section{Correspondence}

Dr. Núria Fabrellas, Professor, School of Nursing, University of Barcelona, Institut d'Investigació Biomèdica de Bellvitge (IDIBELL), Feixa Llarga s/n Pavello de Govern, 3 planta, 08907 L'Hospitalet de Llobregat, Catalonia, spain. E-mail: nfabrellas@ub.edu

Accepted: August 11, 2015

doi: 10.1111/jnu.12169

\section{Abstract}

Introduction: Attention to patients with acute minor illnesses represents a major burden for primary care. Although programs of nurse care for children with acute minor illnesses in primary care started a long time ago, there is limited information about the results of these programs in current practice.

Objectives: The objective of this study was to assess the feasibility and efficacy of a program of nurse management for unscheduled consultations of children with acute minor illnesses.

Methods: Observational study of children seeking unscheduled consultations for 16 acute minor illnesses in 284 primary care practices during a 2year period. The program of nurse management used predefined management algorithms.

Findings: Among 467,160 consultations performed, case resolution was achieved in $65.4 \%$. The remaining $34.6 \%$ of cases were not solved by the primary healthcare nurse due to the existence of signs of alarm and were referred to a pediatrician. Return to consultation during a 7-day period for the same reason as the original consultation was only $2.6 \%$.

Conclusions: A program that uses management algorithms is effective for nurse care management of children with acute minor illnesses in primary care. Clinical Relevance: Application of programs of nurse management for unscheduled consultations for children with acute minor illnesses is feasible and effective.
The increasing demand for unscheduled care of patients with acute minor illnesses represents a challenge for health systems in many countries (Gill, Dawes, Sharpe, 8 Mayou, 1998; Kocher \& Asplin, 2013). Many health systems have therefore developed specific programs to favor the attention of patients with acute minor illnesses in primary care. The ideal system should be timely, accessible, and meet patients' needs and expectations. However, the issue is complex because primary care centers are usually overworked since they have to provide care to an 
increasingly higher number of patients with chronic diseases, often in the setting of a limited number of healthcare professionals (Bodenheimer \& Pham, 2010). As a result, a number of healthcare systems have implemented programs in which the unscheduled acute care of adult patients with minor illnesses is delivered by nurse practitioners. The rationale of these programs is based on a large body of evidence indicating that nurse practitioners are able to provide patients with minor illnesses at a high standard of care similar to that provided by general practitioners. A systematic review of 11 randomized and 23 observational studies comparing nurse practitioners and physicians providing care at the first point of contact in a primary care setting showed no differences in quality of care delivered, as estimated by prescriptions, return consultations, or referrals between the two groups, whereas patients' satisfaction was higher with nurse practitioners (Horrocks, Anderson, \& Salisbury, 2002).

The current study was aimed at assessing the feasibility and efficacy of a program of nurse management for unscheduled consultations of children with acute minor illnesses. Earlier reports of nursing care for children with acute minor illnesses derive from the Pediatric Nurse-Practitioner Program. This was a program developed in the late 1960s at the University of Colorado aimed at providing total health care for children living in medically deprived communities (Silver, Ford, \& Day, 1968; Silver, Ford, \& Stearly, 1967). Nurses provided care to well children as well as sick children with acute or chronic conditions. Children with acute minor illnesses were cared for by nurses using a pre-established plan of management, while children severely ill were referred to a physician for immediate medical care. Although similar programs for children with acute minor illnesses have since then been established, there is limited information on the effectiveness of such programs in current nursing practice (Campbell, Kearsley, Herdman, \& Maric, 1995; Schiff, Fraser, \& Walters, 1969; Yousey \& Carr, 2005). Knowledge about the effectiveness of programs of nurse practitioner management of children with acute minor illnesses is important because acute conditions in the pediatric population are very common and differ from those in adults. In two randomized controlled trials of nurse practitioner care versus general practitioner care for patients requesting same day consultation, 896 out of the 2,660 patients included in the study were children (Kinnerseley, Anderson, Parry, Clement, Archard, \& Turton, 2000; Venning, Durie, Roland, Roberts, \& Leese, 2000). In both studies, patients' satisfaction was higher with nurse practitioners but no other specific analyses were reported for the pediatric population. Although to our knowledge there are no specific studies assessing the frequency of unscheduled consultations in the pediatric population in primary care, they probably represent a major workload for health systems in a manner similar to that for the adult population (Bodenheimer $\&$ Pham, 2010; Kocher \& Asplin, 2013). Due to this limited of information, we report an observational study assessing the results of a program of nurse management for unscheduled consultations of children with acute minor illnesses in primary care in a large geographical area in Catalonia, northwest Spain. The program uses evidence-based algorithms for the most common acute minor illnesses in pediatric patients, including the following: skin injury, acute fever, cough, runny nose, acute diarrhea, diaper rash, vomiting, stomach cramps, burns, bites, constipation, intense crying, ear foreign body, nose bleeding, regurgitation, and nasal foreign body. At the time of the visit, the nurses first assessed the main reason for consultation as well as possible previous health problems and applied the corresponding algorithm. All algorithms had signs of alarm to recognize children with potentially severe conditions that required expedited consultation with a pediatrician. If signs of alarm were not present, nurses applied the algorithm, which specified the cures for each particular condition. An example of an algorithm is provided in Table 1. All algorithms dealt with specific health conditions that had been identified in previous years as prevalent among the pediatric population.

In Spain there is no exact equivalent to the nurse practitioner as in the United Kingdom or United States, but nursing education requires a 4-year university degree. Therefore, although their educational background is lower than that of nurse practitioners in the United States or other countries, it is sufficient to deal with management of patients with minor illnesses. Moreover, with the current legislation, Spanish nurses can prescribe drugs under specific circumstances such as those of predefined algorithms. The results presented herein reflect an expansion of the role of nurses in primary care in Spain.

\section{Methods}

\section{Aim of the Study}

The objective of this study was to assess the feasibility and efficacy of a program of nurse management for unscheduled consultations of children with acute minor illnesses.

\section{Design of the Study}

The current study is an observational study with retrospective analysis of data from children attended in a program of nurse algorithm-guided care for acute minor illnesses in primary care. Data were collected 
Table 1. Nurse Treatment Protocols for Patients With Constipation

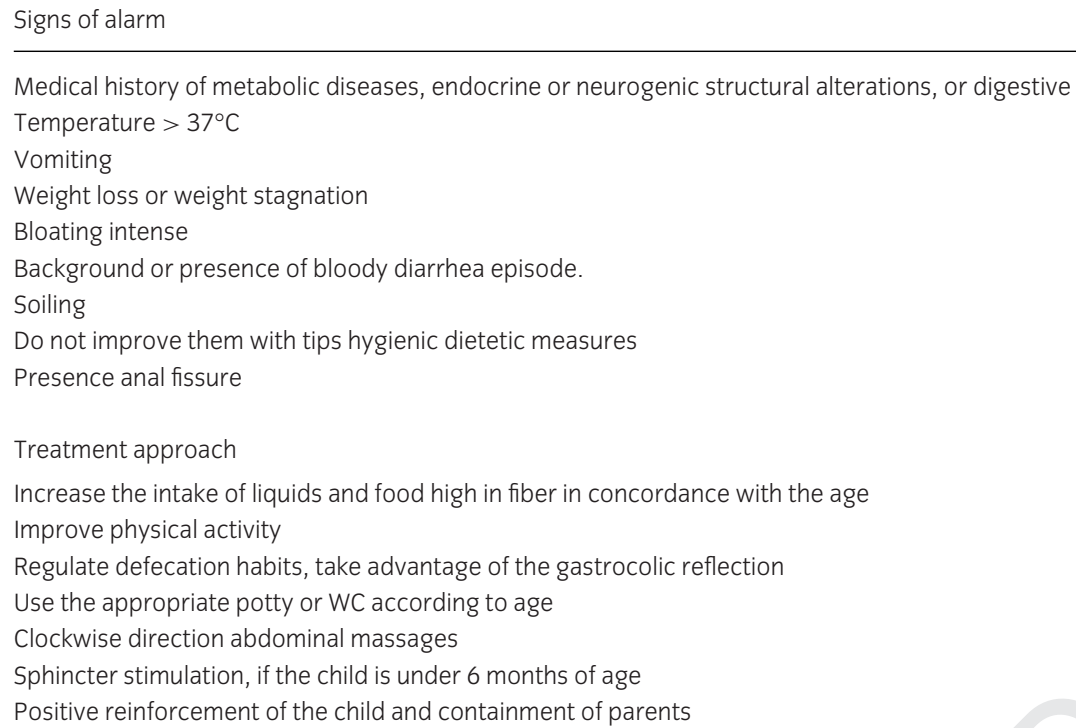

Note. All signs of alarm were looked for in each algorithm. If a sign(s) of alarm was present, the nurses sent the children to the pediatrician for immediate consultation. If none of the signs of alarm was present, the nurses applied the part of the algorithm related to treatment.

prospectively in the electronic medical record at the time of consultation with the child.

\section{Study Population}

A total of 467,160 children up to 15 years old with unscheduled consultations for 16 different acute minor illnesses seen over a 2-year period (April 2009 to April $2011)$ were included in the study.

\section{Program of Nurse Algorithm-Guided Care for Children With Acute Minor Illnesses}

This study was performed in Catalonia, an autonomous region of Spain, located in the northeast corner of the country, next to France, with a population of approximately seven million inhabitants. Catalonia has a public health system with universal coverage. Primary health care is delivered in primary care practices that are widespread around the country in such a way that health care can be provided similarly in small country villages as well as neighborhoods of large cities. All practices have nurses and general practitioners. The role of nurses in primary care is to provide care to patients with chronic illnesses and also patients with acute minor illnesses. Care to children is also provided in primary care centers by pediatricians and nurses trained in pediatrics.

The main healthcare provider in Catalonia launched in 2009 a program of nurse management for patients with acute minor illnesses requesting same-day consultation in primary care. This program included adult patients as well as children. Before the program was implemented, adult patients requesting same-day consultation with acute minor illnesses were seen by general practitioners, while children were seen by pediatricians. These consultations for acute minor illnesses without appointment represented a major workload for both general practitioners and pediatricians. The program was carried out in 284 primary care practices attending a population of approximately 6 million inhabitants.

The pediatric program of nursing care for children with acute minor illnesses was prepared by a task group composed of nurses with extensive experience in pediatrics, pediatricians, and general practitioners, all with experience in primary care. The program was approved by the Institut Català de la Salut (Catalonian Institute of Health), the healthcare provider, and reviewed by nursing and pediatric societies and was in accordance with the current legislation with respect to the role of nurses in patient care. The program consisted of a general protocol and 16 management algorithms of common acute minor illnesses in children seen in primary care, as previously mentioned. Nurse management algorithms were prepared by a group of pediatric nurses and pediatricians following the guidelines of the Institut Català de la Salut for each condition, which were based on available evidence derived from recommendations of national nursing and pediatric societies. Before the implementation 
of the program, working sessions were performed in all practices to explain the management algorithms to all nurses and pediatricians. Moreover, an online training program was specifically designed for further education on the use of the management algorithms. Besides this, as explained below, management algorithms were included in the electronic medical record system used in all practices and could be easily accessed from patients' e-medical records. The general protocol included three steps: assessment of main signs and symptoms, identification of previous health problems and their treatments, and detection of the main reason for consultation. Algorithms included signs of alarm to identify children with a potentially severe condition. If signs of alarm were not present, the nurse applied the algorithm that only in few cases required drug prescription to relieve symptoms (i.e., fever). If signs of alarm were detected, the nurse referred the patient to the pediatrician, who was available for an immediate visit in the same practice. Both nurses and pediatricians advised parents to seek urgent medical assistance if symptoms did not improve or children developed new symptoms. Once the program started, all children seeking same-day consultation for one of the 16 predefined conditions were seen by a nurse in a specific nurse office of primary care practices. In all practices, there was a nurse dealing with these patients 5 days per week. During the consultation, nurses performed a general interview of the children and their parents to identify the main reason for consultation and identify potential previous health issues. Afterwards, nurses applied one of the management algorithms, which were included in the computerized medical system to ensure compliance. All algorithms, besides including instructions for management, incorporated issues of healthcare education and prevention related to each condition. An example of a management algorithm is presented in Table 1. The study was approved by the institutional review board and followed the current regulations for data confidentiality.

\section{Data Collection}

Data from all consecutive children seen in the program during the 2-year period of the study were downloaded directly from the computerized electronic health record system using specific software and incorporated into a database for analysis.

\section{Data Analysis}

The primary outcome was case resolution, defined as application of the management algorithm by the pediatric nurse without the need of referral to the pediatrician. The secondary outcome was return to consultation,
Table 2. Frequency of Acute Minor Illnesses in Pediatric Patients Categorized by Age Evaluated in the Program of Nurse Management in Primary Care Over a 2-Year Period

\begin{tabular}{lcccc}
\hline & $<1$ year & $1-6$ years & $>6$ years & Total (\%) \\
\hline Skin injury & 7,366 & 78,278 & 72,912 & $158,556(33.9)$ \\
Acute fever & 10,626 & 42,424 & 18,812 & $71,862(15.4)$ \\
Cough & 12,662 & 40,428 & 17,196 & $70,286(15.0)$ \\
Runny nose & 18,232 & 20,568 & 7,284 & $46,084(9.9)$ \\
Acute diarrhea & 7,226 & 14,122 & 5,674 & $27,022(5.8)$ \\
Diaper rash & 15,628 & 6,234 & 1,130 & $22,992(4.9)$ \\
Vomiting & 2,222 & 8,278 & 5,202 & $15,702(3.4)$ \\
Stomach cramps & 15,412 & 130 & 130 & $15,672(3.4)$ \\
Burns & 1,368 & 7,884 & 5,412 & $14,664(3.1)$ \\
Bites & 464 & 4,950 & 3,754 & $9,168(2.0)$ \\
Constipation & 3,068 & 1,356 & 362 & $4,786(1.0)$ \\
Intense crying & 1,206 & 654 & 132 & $1,992(0.4)$ \\
Ear foreign body & 56 & 610 & 498 & $1,164(0.3)$ \\
Nose bleeding & 30 & 450 & 508 & $988(0.2)$ \\
Regurgitation & 764 & 24 & 22 & $810(0.2)$ \\
Nasal foreign body & 12 & 382 & 52 & $446(0.1)$ \\
Total & 96,342 & 226,772 & 139,080 & 462,194 \\
& $(20.8 \%)$ & $(49.0 \%)$ & $(30.1 \%)$ & \\
\hline & & & & \\
\hline
\end{tabular}

defined as requirement of new consultation for the same reason as the first one in primary care, either with a pediatric nurse or pediatrician, within a 7-day period after the initial consultation. In the calculation of the rate of return to consultation, only cases that were solved by nurses were included in the analysis. Comparisons of resolution rates and return consultation between the different age groups of children were performed with the chi-square test and Fisher's exact test. The analysis was performed using SPSS 15 for Windows (SPSS, Inc., Chicago, IL, USA).

\section{Findings}

Frequency of Unscheduled Acute Minor Illnesses in Children During the 2-year period of the study, over 450,000 unscheduled consultations of 16 predefined acute minor illnesses in children were performed by pediatric nurses under the current program using nurse management algorithms in primary care. This represents approximately 19,000 consultations per month in all practices, and an average of 68 consultations per practice per month.

The 16 acute minor illnesses and their individual frequencies categorized by age groups are shown in Table 2.

\section{Case Resolution}

Overall case resolution was achieved in almost two thirds of children $(65.4 \%)$. The remaining $34.6 \%$ of cases 
Table 3. Case Resolution (in Percentage) of Acute Minor Illnesses in Pediatric Patients Categorized by Age Evaluated in the Program of Nurse Management in Primary Care Over a 2-Year Period

\begin{tabular}{lccccc}
\hline & $<1$ year & 1 -6years & $>6$ years & Total & $p$ value \\
\hline Stomach cramps & 96.9 & 83.0 & 90.0 & 96.8 & $<.0001$ \\
Burns & 91.6 & 94.0 & 96.0 & 94.9 & $<.0001$ \\
Diaper rash & 90.0 & 86.0 & 84.0 & 89.0 & $<.0001$ \\
Regurgitation & 89.7 & 66.0 & 72.0 & 88.6 & $<.0001$ \\
Constipation & 91.0 & 83.0 & 76.0 & 87.8 & $<.0001$ \\
Skin injury & 87.0 & 86.5 & 87.0 & 87.1 & $<.0001$ \\
Nose bleeding & 60.0 & 71.0 & 75.5 & 73.1 & .0773 \\
Ear foreign body & 67.8 & 70.0 & 75.0 & 72.5 & .0754 \\
Nasal foreign body & 100 & 68.0 & 76.9 & 70.4 & .0349 \\
Acute diarrhea & 65.0 & 67.0 & 72.0 & 68.0 & $<.0001$ \\
Bites & 79.0 & 66.0 & 65.0 & 66.4 & $<.0001$ \\
Runny nose & 66.0 & 56.9 & 62.0 & 61.6 & $<.0001$ \\
Vomiting & 43.0 & 52.0 & 55.0 & 52.2 & $<.0001$ \\
Intense crying & 52.0 & 38.0 & 48.0 & 47.4 & $<.0001$ \\
Cough & 28.7 & 34.0 & 39.6 & 34.7 & $<.0001$ \\
Acute fever & 27.8 & 28.0 & 35.0 & 30.0 & $<.0001$ \\
Average & 68.2 & 60.8 & 71.0 & 65.4 & $<.001$ \\
\hline & & & & & \\
\hline
\end{tabular}

were not solved by the pediatric nurse due to the existence of predefined signs of alarm and were referred to a pediatrician. The individual frequencies of case resolution varied widely according to the different conditions, from $96.8 \%$ for stomach cramps to $30 \%$ for acute fever (Table 3). Resolution rates of over $80 \%$ were achieved for stomach cramps, burns, diaper rash, regurgitation, constipation, and skin injury. Resolution rates of $50 \%$ to $80 \%$ were obtained for nose bleeding, ear and nasal foreign bodies, acute diarrhea, bites, runny nose, and vomiting. The lowest resolution rates (below 50\%) were found for intense crying, cough, and acute fever. Overall, the resolution rate was significantly lower in the 1 - to 6-year age group compared to the other two groups $(p<.001)$.

\section{Return to Consultation}

In the whole series, the proportion of children who returned to consultation was low and averaged only $2.6 \%$. The highest return rates were observed for burns and skin injury and the lowest for nasal foreign body $(9.8 \%$ and $3.3 \%$ vs. $0 \%$, respectively; Table 4 ). The return rate was lower than $3 \%$ in 14 out of the 16 acute minor illnesses. Considering all conditions together, return to consultation increased slightly but significantly with age, from $1.6 \%$ in the age group lower than 1 year, $2.6 \%$ in the age group 1 to 6 years, and $3.2 \%$ in the age group more than 6 years $(p<.001)$.
Table 4. Return to Consultation (in Percentage) of Acute Minor Illnesses in Pediatric Patients Categorized by Age Evaluated in the Program of Nurse Management in Primary Care Over a 2-Year Period

\begin{tabular}{lccccc}
\hline & $<$ year & $1-6$ years & $>$ 6 years & Total & $p$ value \\
\hline Burns & 10.8 & 10.2 & 9.0 & 9.8 & .0324 \\
Skin injury & 2.9 & 2.9 & 3.7 & 3.3 & $<.0001$ \\
Acute fever & 2.6 & 2.2 & 1.7 & 2.1 & .0101 \\
Cough & 2.2 & 1.6 & 1.2 & 1.6 & .0002 \\
Constipation & 1.5 & 0.8 & 3.6 & 1.3 & .0032 \\
Nose bleeding & 0 & 0.6 & 1.5 & 1.1 & .4471 \\
Acute diarrhea & 1.8 & 1.0 & 0.5 & 1.1 & $<.0001$ \\
Runny nose & 1.3 & 0.6 & 0.4 & 0.9 & $<.0001$ \\
Vomiting & 2.3 & 0.9 & 0.4 & 0.9 & $<.0001$ \\
Stomach cramps & 0.8 & 0 & 0 & 0.8 & .3985 \\
Regurgitation & 0.8 & 0 & 0 & 0.8 & .8694 \\
Bites & 0.5 & 0.6 & 0.4 & 0.6 & .6637 \\
Diaper rash & 0.5 & 0.5 & 1.4 & 0.6 & .0017 \\
Ear foreign body & 0 & 0.9 & 0 & 0.5 & .1416 \\
Intense crying & 0.3 & 0 & 0 & 0.2 & .6065 \\
Nasal foreign body & 0 & 0 & 0 & 0.0 & 1 \\
Average & 1.5 & 2.6 & 3.2 & 2.6 & $<.001$ \\
\hline & & & & & \\
\hline
\end{tabular}

\section{Discussion}

The results of the current study show that a program of nurse management for unscheduled consultations of children is feasible and effective in a large primary care organization. The results of the current study should be discussed in the light of the progressively increasing role of nurses in primary care. Advanced nurse practitioners are expanding their role in primary care in many countries, including the United States, Canada, European countries, Australia, and New Zealand, among others, to address the increased demand related to the growing number of patients with chronic disorders as well as patients with acute uncomplicated disorders (Bodenheimer, Chen, \& Bennett, 2009; Fairman, Rowe, Hassmiller, \& Shalala, 2011). This expansion of the role of advanced nurse practitioners is not free of controversy, which has been discussed in several studies reported elsewhere (Donelan, DesRoches, Dittus, \& Buerhaus, 2013; Iglehart, 2013; Lindblad, Hallman, Gillsjö, Lindblad, \& Fegerström, 2010; Vallejo-Torres \& Morris, 2011). The most contentious aspects of the expansion of the role of nurses are the level of education required by nurses, drug prescription, and the issue of supervision by a physician. Moreover, comparisons of levels of nurse education and training between countries are difficult because of differences in educational programs and qualifications among countries.

In the program reported in the current study, all nurses who participated had a university degree followed by experience with pediatric patients. In the current program, 
the only drugs that could be prescribed were drugs to relieve symptoms (i.e., fever, cough), and prescription was performed according to management algorithms. Finally, there was a close coordination between nurses and pediatricians, who were always available in each practice for consultation.

A number of previous experiences about nursing care of children with acute minor illnesses have been reported (Campbell et al., 1995; Schiff et al., 1969; Silver et al., 1967, 1968; Yousey \& Carr, 2005). The current study provides a further insight in this field by analyzing the efficacy of one of these programs in current practice in a heavily populated area. The most frequent conditions seen were skin injury, acute fever, cough, runny nose, and acute diarrhea, which accounted for approximately $80 \%$ of the total consultations. These conditions are similar to those reported by others (Whitburn et al., 2011). Individual frequencies of the remaining conditions were lower than $5 \%$. The highest resolution rates, over $80 \%$, were achieved with stomach cramps, burns, diaper rash, regurgitation, constipation, and skin injury. The lowest rates were achieved in cough and acute fever $34.7 \%$ and $30 \%$, respectively). The difference in resolution rates may be due, at least in part, to the fact that the former categories include conditions related to classical nurse practice, while the latter require exploratory maneuvers that may be less familiar to nurses. Moreover, it should be emphasized that algorithms for cough and fever included several signs of alarm aimed at transferring children to the pediatrician. Therefore, the lower resolution rate was likely related to the strict adherence to management algorithms. The overall effectiveness of the program was confirmed by the low rate of return consultation for the majority of conditions, lower than $2 \%$ for all but two conditions, skin injury and burns $(3.3 \%$ and $9.8 \%$, respectively).

It should be emphasized that the nurse program for unscheduled acute care in children reported in this study was based on management algorithms incorporated in the electronic medical record system and that training sessions were performed before the program started. This allowed applying updated and evidence-based diagnostic and management processes and eliminating inconsistencies across nurses and practices. A further point to highlight is that a pediatrician was always available for immediate consultation in cases where the management algorithms showed signs of alarm. Therefore, the program was an example of close collaboration between nurses and physicians. Our group has reported similar results with a nurse program for same-day management of acute minor illnesses in adult patients (Fabrellas et al., $2011,2013)$.

\section{Limitations of the Study}

This study has several limitations that should be mentioned. First, the protocol developed may not be entirely applicable to countries with health systems different from the current system in Catalonia and may need some adjustments to comply with local particularities and regulations. Second, the program reported requires a close collaboration between nurses and pediatricians both in the development of algorithms and during its application. Without this collaboration the program cannot be developed. Moreover, the current program requires nurses with a university degree and significant experience in pediatrics. Third, the study was based on retrospective analysis of data; therefore, it has the limitations inherent to all retrospective studies. For instance, case resolution was not confirmed directly with parents or children and patient satisfaction could not be analyzed.

\section{Further Research}

Possible further research in this area includes the development of new algorithms for conditions not included in the current program as well as the potential evaluation of a telephone nursing line for assistance of simple conditions.

In summary, the current study reports the results of a large program of nurse management for unscheduled consultations of children with acute minor illnesses in primary care. The program was feasible and proved to be very effective in case resolution. The establishment of this type of program may help nurses expand their role in primary care and appears to be an efficient strategy to cope with the increased demand for acute unscheduled care.

\section{Acknowledgments}

The authors would like to acknowledge the work of nurses of the same day consultation program of all practices of the Institut Català de la Salut.

\section{Clinical Resources}

- Improving NHS children's community nursing services: https://www.gov.uk/government/ publications/nhs-at-home-childrens-communitynursing-services

- National Ambulatory Medical Care Survey and National Hospital Ambulatory Medical Care Survey, 2007. National Center for Health Statistics, 2010. https://www.cdc.gov/nchs/ahcd/ahcd_reports.htm 


\section{References}

Bodenheimer, T., Chen E., \& Bennett H. G. (2009). Confronting the growing burden of chronic disease: Can the U.S. health care workforce do the job? Health Affairs, 28, 64-74. doi:10.1377/hlthaff.28.1.64

Bodenheimer, T., \& Pham, H. H. (2010). Primary care: Current problems and proposed solutions. Health Affairs, 29, 799-805. doi:10.1377/hlthaff.2010.0026

Campbell, A., Kearsley, N., Herdman, M., \& Maric, S. (1995). Establishing a minor illness nurse in a busy general practice. May reduce doctors' workload. British Medical Journal, 27, 1404-1405.

Donelan, K., DesRoches, C. M., Dittus, R. S., \& Buerhaus, P. (2013). Perspectives of physicians and nurse practitioners on primary care practice. New England Journal of Medicine, 368, 1898-1906. doi:10.1056/NEJMsa 1212938

Fabrellas, N., Sánchez, C., Juvé, E., Aurin, E., Monserrat, D., Casanovas, E., \& Urrea, M. A. (2013). Program of nurse algorithm-guided care for adult patients with acute minor illnesses in primary care. BMC Family Practice, 14, 61. doi:10.1186/1471-2296-14-61

Fabrellas, N., Vidal, A., Amat, G., Lejardi, Y., Deulofeu, M. P., \& Buendia, C. (2011). Nurse management of "same day" consultation for patients with minor /illnesses: Results of an extended programme in primary care in Catalonia. Journal of Advanced Nursing, 67, 1811-1816. doi:10.1111/j.1365-2648.2011.05624.x

Fairman, J. A., Rowe, J. W., Hassmiller, S., \& Shalala, D. E. (2011). Broadening the scope of nursing practice. New England Journal of Medicine, 364, 193-195. doi:10.1056/NEJMp1012121

Gill, D., Dawes, M., Sharpe, M., \& Mayou, R. (1998). GP frequent consulters: Their prevalence, natural history, and contribution to rising workload. British Journal of General Practice, 48, 1856-1857.

Horrocks, S., Anderson, E., \& Salisbury, C. (2002). Systematic review of whether nurse practitioners working in primary care can provide equivalent care to doctors. British Medical Journal, 324, 819-823. doi:10.1136/bmj.324.7341.819

Iglehart, J. K. (2013). Expanding the role of advanced nurse practitioners-Risks and rewards. New England Journal of Medicine, 368, 1935-1941. doi:10.1056/NEJMhpr1301084
Kinnerseley, P., Anderson E., Parry, K., Clement, J., Archard, L., \& Turton, P. (2000). Randomised controlled trial of nurse practitioner versus general practitioner care for patients requesting "same day" consultations in primary care. British Medical Journal, 320, 1043-1048.

Kocher, K. E., \& Asplin, B. R. (2013). What is our plan for acute unscheduled care? Annals of Internal Medicine, 158, 907-909. doi:10.7326/0003-4819-158-12-201306180-00008

Lindblad, E., Hallman, E. B., Gillsjö, C., Lindblad, U., \& Fegerström, L. (2010). Experiences of the new role of advanced practice RN in Swedish primary health care-A qualitative study. International Journal of Nursing Practice, 16, 69-74. doi:10.1111/j.1440-172X.2009.01810.x

Schiff, D. W., Fraser, C. H., \& Walters, H. L. (1969). The pediatric nurse practitioner in the office of pediatricians in private practice. Pediatrics, 44, 62-68.

Silver, H. K., Ford, L. C., \& Day, L. R. (1968). The pediatric nurse-practitioner program. Expanding the role of the nurse to provide increased health care for children. Journal of the American Medical Association, 204, 88-92. increase health care for children: The pediatric nurse practitioner program. Pediatrics, 39, 756-760.

Vallejo-Torres, L., \& Morris, S. (2011). Factors associated with the use of primary care services: The role of practice nurses. European Journal of Health Economics, 12, 373-381. doi:10.1007/s10198-010-0251-5

Venning, P., Durie, A., Roland, M., Roberts, C., \& Leese, B. (2000). Randomised controlled trial comparing cost effectiveness of GP and nurse practitioners in primary care. British Medical Journal, 320, 1048-1053.

Whitburn, S., Costelloe, C., Montgomery, A. A., Redmond, N. M., Fletcher, M., Peters, T. J., \& Hay, A. D. (2011). The frequency distribution of presenting symptoms in children aged six months to six years to primary care. Primary Health Care and Development, 12, 123-134. doi:10.1017/S146342361000040x

Yousey, Y., \& Carr, M. (2005). A health care program for homeless children using Healthy People 2010 objectives. Nursing Clinics of North America, 40, 791-801. 


\section{Author Queries}

Q1: Au: Please confirm that given names (red) and surnames/family names (green) have been identified correctly.

Q2: AU: Please supply job title for Torres.

Q3: $\mathrm{Au}$ : Is there a word missing after "digestive"?

Q4: Au: Meaning of "tips" unclear here.

Q5: Au: Meaning unclear. Do you mean to say "the words 'potty' or 'WC"'?

Q6: $\mathrm{Au}$ : "rate to return to consultation" was changed to "rate of return to consultation." Please verify that your meaning has been preserved.

Q7: Au: Please verify the correctness of all URLs and dois given in this paper.

Q8: Au: For Fabrellas et al. (2013), please provide the page range, unless this is a one-page article.

Q9: Au: "Shifff" was changed to "Schiff" both here an in in-text citations. Please verify this spelling.

Q10: Au: "Fords" was changed to "Ford" both here and in in-text citations. Please verify this spelling in both Silver et al. reference entries. 